\title{
Editorial
}

\section{Nanoceramics: Synthesis, Characterization, and Applications}

\author{
Amirkianoosh Kiani, ${ }^{1}$ Mohsen Rahmani, ${ }^{2}$ Sivakumar Manickam, ${ }^{3}$ and Bo Tan ${ }^{4}$ \\ ${ }^{1}$ Department of Mechanical Engineering, University of New Brunswick (UNB), Canada \\ ${ }^{2}$ Department of Physics (EXSS), Imperial College London, 901 Blackett Lab, Prince Consort Road, London, UK \\ ${ }^{3}$ Department of Sciences, Amrita School of Engineering, Amrita Vishwa Vidyapeetham, Amritanagar (PO), \\ Coimbatore, Tamil Nadu, India \\ ${ }^{4}$ Department of Aerospace Engineering, Ryerson University, Canada
}

Correspondence should be addressed to Bo Tan; tanbo@ryerson.ca

Received 11 June 2014; Accepted 11 June 2014; Published 26 June 2014

Copyright (C) 2014 Amirkianoosh Kiani et al. This is an open access article distributed under the Creative Commons Attribution License, which permits unrestricted use, distribution, and reproduction in any medium, provided the original work is properly cited.

Over the past decade, nanoceramics have received significant attention as candidate materials due to their capability to demonstrate improved and unique properties in comparison with conventional bulk ceramic materials. Nanoceramics exhibit unique processing, mechanical, and surface characteristics such as superplasticity, machinability, strength, toughness, and bioactivity due to the fine grain size, abundant grain boundaries, and controllable crystallinity.

This issue compiles five exciting manuscripts, which address recent trends and development in nanoceramics.

The optical properties of nanoceramics are addressed in three manuscripts. Optical nonlinear performance of silicon nanoparticles at different doping concentrations has been investigated by $\mathrm{L}$. Chen et al. Their results show silicon nanoparticles generated by femtosecond laser ablation exhibit better saturable absorption performance at higher doping concentrations. Their results reveal the possibility to tune the optical nonlinearity of silicon nanoparticles by changing the doping concentration.

In recent times, there are great interests in luminescent materials for efficient frequency conversion from infrared to visible radiation; oxyfluoride glass ceramics are ambivalent materials which can exhibit optical properties of fluoride single-crystals when they are doped with rare-earth ions. M. $\mathrm{H}$. Imanieh et al's manuscript focuses on improvement of $\mathrm{Er}^{3+}$ emissions in two series of oxyfluoride glass ceramics $\left(\mathrm{SiO}_{2} / \mathrm{Al}_{2} \mathrm{O}_{3}\right.$ and $\left.\mathrm{SiO}_{2} / \mathrm{Al}_{2} \mathrm{O}\right)$ containing $\mathrm{CaF}_{2}$ nanocrystals doped with a fixed amount of $\mathrm{Er}^{3+}$ and $\mathrm{Yb}^{3+}$ through the heat treatment at different temperatures. They showed in their study that increasing the temperature of the heat treatment leads to a rise in the red and green emissions in the upconversion luminescence of the treated samples. Also, increasing the heat treatment temperature leads to the incorporation of $\mathrm{Er}^{3+}$ ions into $\mathrm{CaF}_{2}$ crystals and can increase the $\mathrm{Yb}^{3+}$ concentration. Increased $\mathrm{Yb}^{+3}$ concentration improves the energy transfer and back transfer process between $\mathrm{Er}^{3+}$ and $\mathrm{Yb}^{3+}$ ions and as a result upconversion intensity can be increased.

Polymers have attracted a lot of attention as excellent host materials for encapsulation of metal nanoparticles like silver, gold, copper, and so forth. Many reports in the literature show attempts for synthesis of metal-polymer nanocomposites based on polymers, with the possibility of variation in their optical, mechanical, and electrical properties for the application in photovoltaic and biomedical devices fabrication. M. Ghanipour et al. investigated the effect of silver nanoparticles doped in PVA on the structural and optical properties of composite films. Their results show that by embedding silver nanoparticles inside the polymer, a number of Bragg's planes in the structure of polymer and its crystallinity are increased noticeably. Ag-O bonds are formed in the films and the bandgap energy, refractive index, and dielectric constant of samples are decreased by increasing the concentrations of silver nanoparticles.

Two of the manuscripts deal with fabrication methods for high performance nanoceramics and nanomaterials; $Q$. Liu et 
al. reported the new method for fabrication of highly ordered $\mathrm{Ti}-\mathrm{Nb}-\mathrm{Zr}-\mathrm{O}$ nanotube arrays through pulse anodic oxidation of $\mathrm{Ti}-\mathrm{Nb}-\mathrm{Zr}$ alloy in monosodium phosphate containing $0.5 \mathrm{wt} \% \mathrm{HF}$ electrolytes. The effect of anodization parameters and $\mathrm{Zr}$ content on the microstructure and composition of $\mathrm{Ti}$ $\mathrm{Nb}-\mathrm{Zr}-\mathrm{O}$ nanotubes have been studied using experimental analysis and it has been found that length of the Ti-Nb-Zr$\mathrm{O}$ nanotubes increased with increase of $\mathrm{Zr}$ contents.

Y. Qiang et al. investigated the materials and chemical properties of BCFN dense ceramic membrane with submicron-CYDC porous layer by the partial oxidation of coke oven gas (COG) in hydrogen production. The results of their study show that this structure exhibits higher stability and no chemical reaction at high temperature environment. Also, the influences of YDC modification on the surface kinetics and oxygen permeation rates of BCFN membranes have been analyzed.

By compiling these papers, we hope to enrich our readers and researchers with respect to synthesis, characterization, and applications of nanoceramics.

\section{Acknowledgments}

Finally, we would like to express our gratitude to the many reviewers for their hard works. We would also like to thank the authors for their contributions to the special issue. This special issue could not have been completed without their dedication and support.

Amirkianoosh Kiani Mohsen Rahmani

Sivakumar Manickam

Bo Tan 

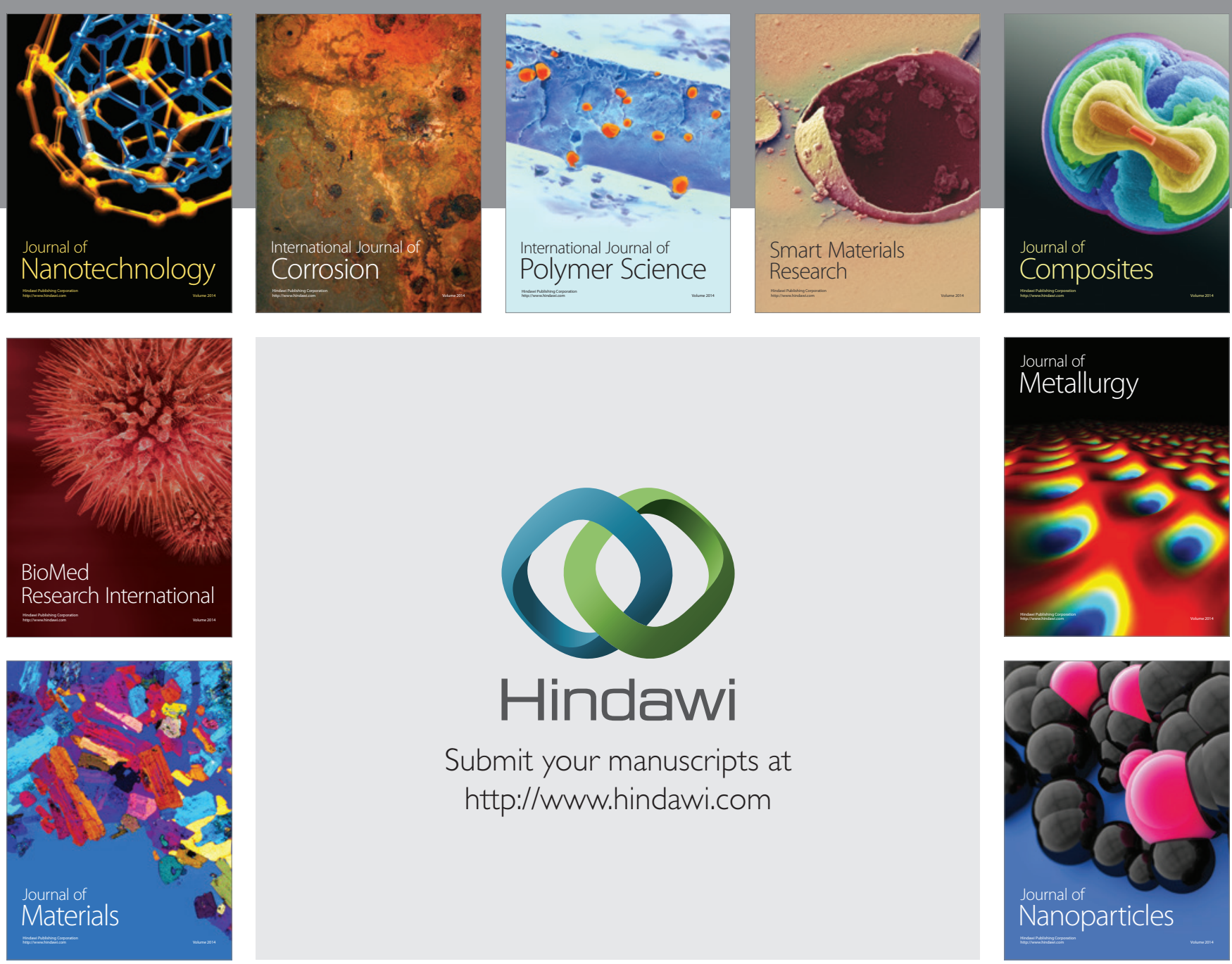

Submit your manuscripts at http://www.hindawi.com
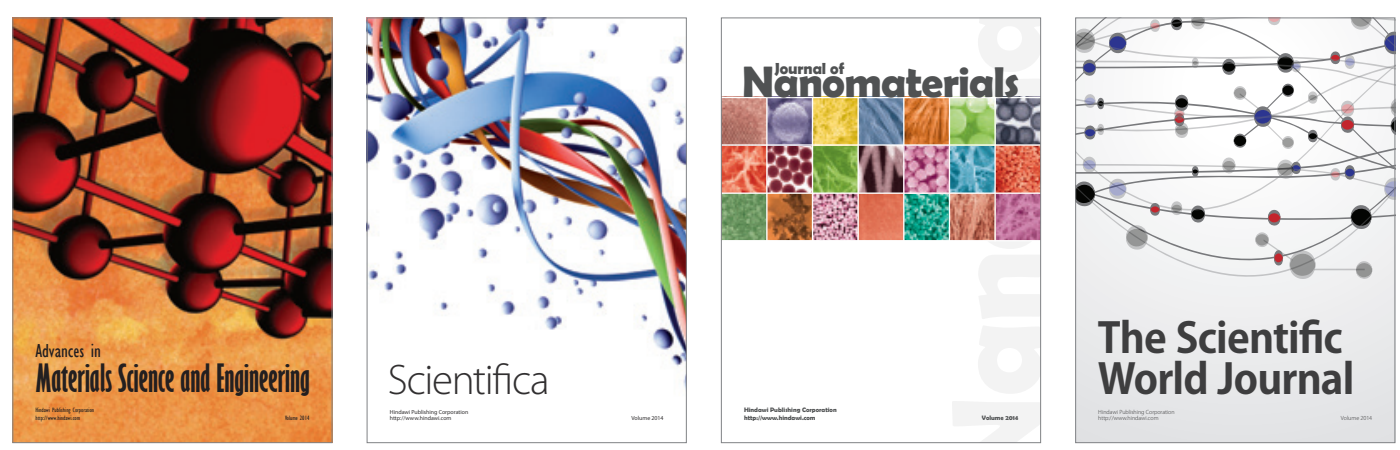

\section{The Scientific World Journal}
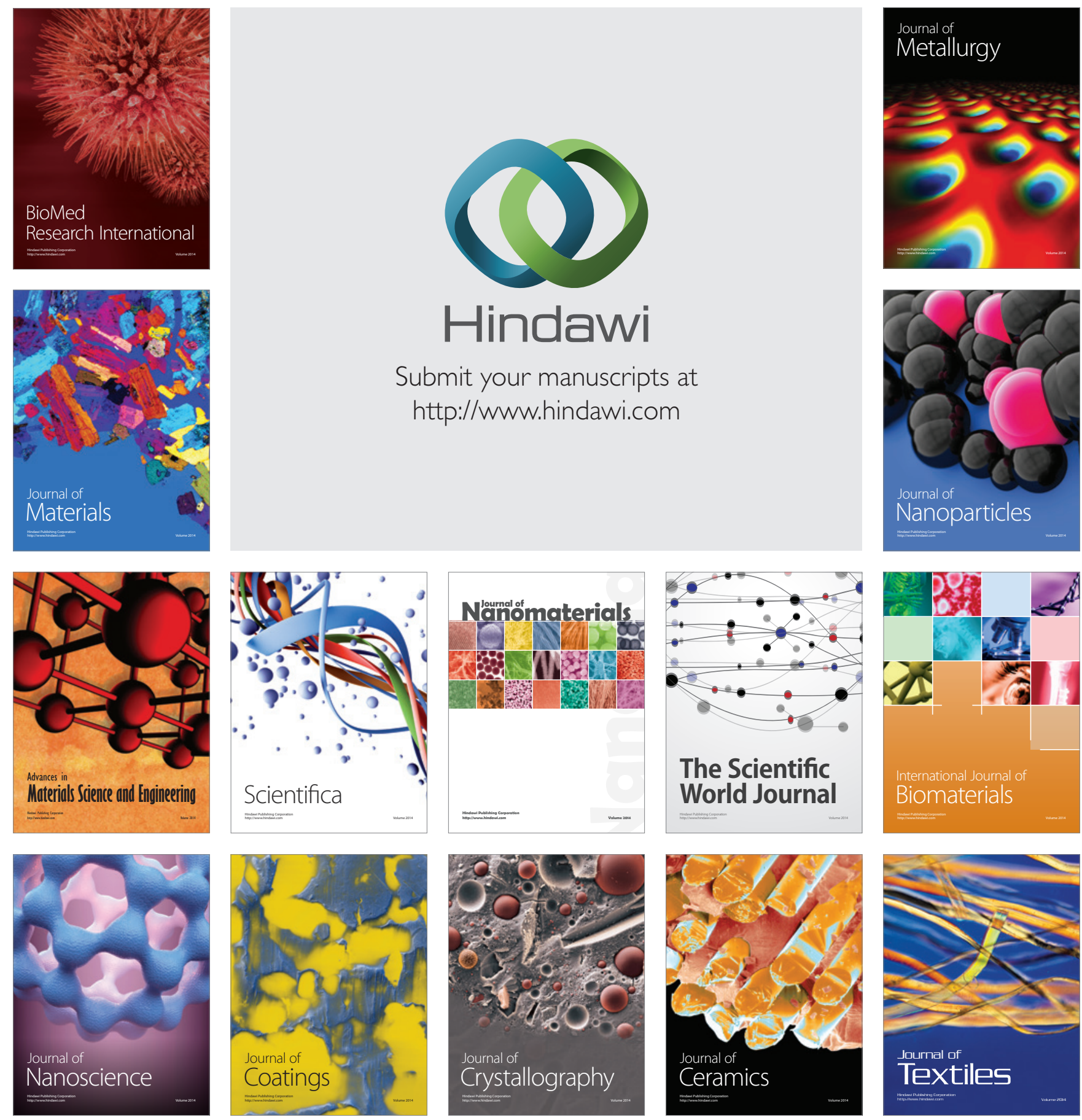